\title{
A Study of Hydraulically Actuated Oscillating Pitch to Increase Energy Production in Wind Turbines
}

\author{
Daniel Escobar, John Sampson, and Kim A. Stelson
Mechanical Engineering Department, University of Minnesota, Minneapolis, Minnesota/United States of America
E-mail: escob060@umn.edu, samps251@umn.edu,kstelson@umn.edu

\begin{abstract}
The focus of this paper is to oscillate the pitch of the wind turbine blades using hydraulic actuation to increase the power captured by a hydrostatic wind turbine. This is based on the fact that oscillating an airfoil under specific conditions increases the instantaneous lift coefficient by up to 97\%. Different conditions were investigated by varying airfoil shape, waveform shape, waveform amplitude, waveform frequency and average angle of attack. The following conditions were found to give the best performance: a low camber airfoil, a lower average angle of attack than used for a non-oscillating airfoil, and an optimized waveform shape called a "tilted sinusoid." A multi-level factorial experiment determined the most impactful variables were frequency, amplitude, and average angle of the tilted sinusoid. The best frequencies and amplitudes were studied through numerical simulations and hill climbing optimization. Results showed that oscillating the pitch of the wind turbine blades would, under all conditions tested, never outperform steady-state turbine operation, the main reason being the higher drag coefficients that are also present when oscillating the pitch of the blade.
\end{abstract}

Keywords: Hydrostatic Transmission, Wind Power, Pitch Oscillation, Energy Storage, Accumulator.

\section{Introduction}

Wind energy is the fastest growing energy source across the world. As climate change and irreversible environmental damage rises, wind energy is seen as a clean alternative to greenhouse gas emitting fossil fuels. It currently accounts for $6.3 \%$ of U.S. energy production and $5 \%$ of energy production worldwide [1]. Wind energy will continue to grow rapidly in the next decade. The Department of Energy (DOE) had set the gal for wind energy to comprise $10 \%$ of domestic power production by $2020,20 \%$ by 2030 and $35 \%$ by 2050 [2]. Although we missed the goal for 2020, we are confident that we will greatly exceed the goal for 2030 and that we will achieve the goal for 2050 because of the exponential growth trend of wind power production.

There are many challenges associated with wind energy production and a major one is its energy efficiency. Most wind turbines capture roughly 0.35 to 0.45 of the energy in the wind, a ratio known as the power coefficient, $C_{p}$. A well-known theory developed in 1919 by Albert Betz sets the theoretical maximum limit of $C_{p}$ as 0.593 [3], [4]. The goal of our wind turbine research is to reduce losses in wind turbines and raise efficiencies to be as close to this value as possible by implementing hydraulic systems and advanced controls. Increasing the efficiency of wind turbines will reduce the overall cost of wind energy and further increase its implementation across the world.

Wind turbine controls include torque, pitch and yaw control and they operate in four control regions, fig. 1. In our research we focus on torque control and pitch control. Conventionally, torque control is used to maximize the power in region 2 and pitch control is used to regulate the power in region 3 . The control strategy in regions 1 and 4 consists of stopping the operation of the turbine because of low wind speeds in region 1 or damaging high wind speeds in region 4. 


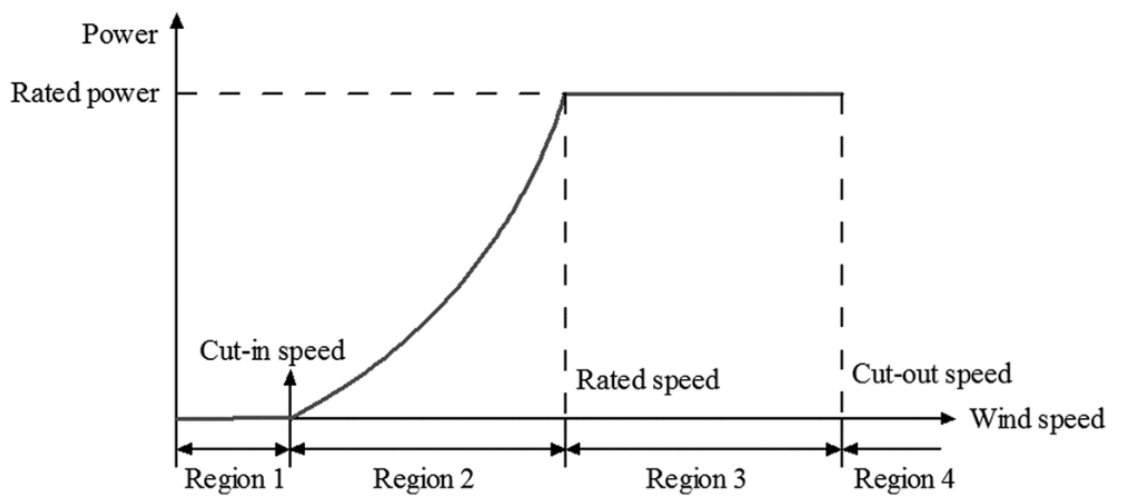

Figure 1: Control regions of a conventional wind turbine [5]

Most research approaches for improving power capture of wind turbines consist of maximum power point tracking (MPPT) in region 2. MPPT is achieved most successfully by controlling the rotor torque. Multiple approaches have been proposed and are summarized in [6] - [8]. Other approaches such as Model Predictive Control [9], and Extremum Seeking Control [10] have been proposed and also show promising results. These approaches are exclusive for traditional fixed-gearbox turbine transmissions, leaving an opportunity for researchers to investigate the performance of hydraulic transmissions for wind turbines.

The focus of our research is on hydraulic transmissions for midsize wind turbines (100 kW to $1 \mathrm{MW}$ ). In this sector, hydrostatic transmissions (HSTs) have many benefits over traditional fixed-gearbox turbine transmissions. For example, a higher power to weight ratio, an infinitely variable transmission ratio, and the ability to run without the need for costly power electronics [11], [12]. Also, there are off-the-shelf components that are readily available to use in this power level. Conventional gearboxes are prone to fatigue damage [13] whereas hydraulic components are not. Previous research from our group has shown that the overall efficiency of an HST turbine is comparable to a gearbox turbine [14]. Taking advantage of the hydraulics in the HST turbine, we have explored different options for improving the efficiency of the wind turbine. We have explored a hybridized HST that uses an accumulator and a secondary pump to store energy in region 3 and release it in region 2 improving the overall performance of the turbine by 4\% [5], [15]. We are currently modifying an HST test stand at the University of Minnesota to experimentally validate the idea.

Oscillating the pitch to increase the lift forces and the torque on the rotor to generate more power has also been explored. Oscillating an airfoil generates a phenomenon of delayed flow separation known as dynamic stall [16], which, under very specific conditions (frequency, amplitude and average angle of attack) can increase the instantaneous maximum lift coefficient by up to $97 \%$ [17]. This concept is well described in the literature, especially for load and aeroelastic calculations. Oscillating pitch models were originally developed for helicopter applications and have been adapted for wind turbine modelling [18]. Holierhoek et al. [19] did a systematic comparison between experimental data and three of the most used models on wind turbine airfoils, but never explored the opportunity to improve power harvesting. In this study we explored through computational fluid dynamics (CFD) simulations and Blade Element Momentum Theory (BEMT) the possibility of using oscillating pitch for wind turbine applications. The paper will present the tools and methods used to perform the study and will later explain the different results obtained through simulations and optimization.

\section{Design and Modeling}

\subsection{Blade Element Momentum Theory (BEMT)}

A turbine blade has an airfoil cross-section with continuously varying chord, orientation and shape along its length as shown in fig. 2. The variation from root to mid span to tip is required so that the blade is strong and efficient. The blade is twisted so that the angle of attack is constant along its length. The product of the chord length and the radius is designed to be constant so that the Reynolds number is constant. For this reason, the chord length decreases with radius. 


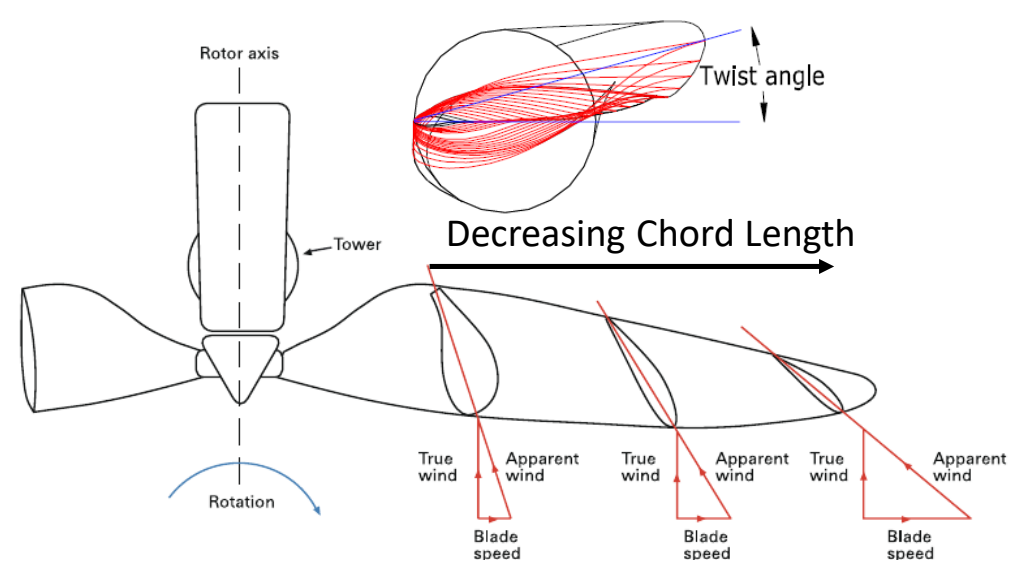

Figure 2: A typical modern horizontal axis wind turbine blade with airfoil profiles, twist and chord length variation [20]

The importance of the aforementioned aspects of wind turbine blade design is that they allow for characterization of the full 3D turbine blade from the physics of a single 2D cross-section using BEMT. BEMT is a combination of momentum theory and blade element theory. Momentum theory by itself analyses the momentum balance of the rotating annular stream tube passing through the turbine, fig. 3 [21]. Blade element theory is the study of the forces generated by the airfoil's lift and drag forces, fig. 4, at different sections along the length of the blade [22]. The combination of both allows us to obtain useful relationships that lead towards fast and simple calculations. The method assumes steady wind conditions, no interaction in between elements, nor wake expansion. Methods to include tip losses, yaw of the turbine, 3D corrections among others have been implemented to improve the analytical results [23], but are not included in this work.

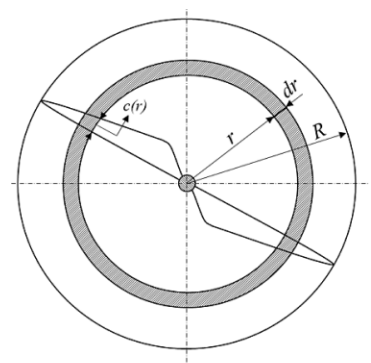

(a)

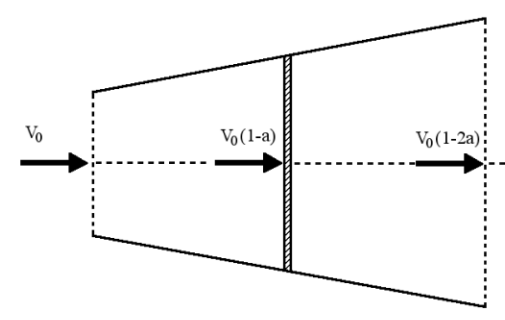

(b)

Figure 3: Typical geometry used for BEM theory analysis (a) Actuator disk (b) Fluid stream tube [23]

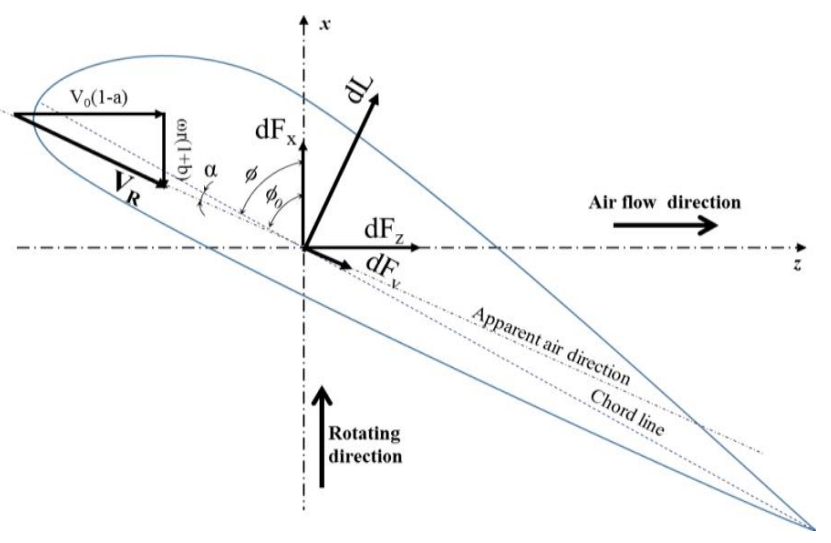

Figure 4: Forces acting on the single blade element [23]

From BEMT we can calculate the torque generated by each element of the wind turbine blade using eq. (1),

$$
d T=\frac{1}{2} \rho B c(r) R(r) V_{R}^{2}\left(C_{L} \sin (\phi)-C_{D} \cos (\phi)\right) d r
$$


Where $\rho$ is the density of the air, $B$ is the number of blades, $c(r)$ is the chord length of the element at distance from the hub $r, R(r)$ is the radius of the blade at distance from the hub $r, V_{R}$ is the relative velocity of the wind, $C_{L}$ is the aerodynamic lift coefficient, $C_{d}$ is the aerodynamic drag coefficient, and $\phi$ is the angle of relative velocity of the wind with respect to the axis of the rotor. The total power generated by the turbine can then be calculated using eq. (2),

$$
P_{r}=\int_{R_{h u b}}^{R} \omega d T
$$

where $R_{h u b}$ is the radius of the turbine hub, $R$ is the radius of the blade, and $\omega$ is the rotational velocity of the blades.

The net power for the static case is the power generated by the blades on the rotor, eq. (2). The net power for the blade oscillation case is the power generated by the oscillating blades minus the power needed to oscillate the blades, $P_{o s c}$, eq. (3). To calculate $P_{o s c}$ we assume steady-state operation and no friction losses,

$$
P_{\text {osc }}=\omega_{p} \tau_{\text {aero }}
$$

Where $\omega_{p}$ is the rotational velocity of the pitching and $\tau_{\text {aero }}$ is the torque generated on the blade by the aerodynamic forces and can be calculated by,

$$
\tau_{\text {aero }}=\frac{1}{2} C_{M_{\text {osc }}} \rho V_{R}^{2} c(r)^{2} d r
$$

and $C_{M_{O S C}}$ is the aerodynamic moment coefficient of the oscillating blade. The dynamic power in the rotor is,

$$
P_{d y n}=P_{r}-P_{o s c}
$$

Taking into account that $C_{L}$ and $C_{D}$ in eq. (1) are the coefficients of the oscillating blade.

\subsection{Variable Selection}

To evaluate the performance of the oscillating pitch versus the static pitch a response variable, the power ratio, $P_{\text {ratio }}$, is introduced. The equation for the power ratio is,

$$
P_{\text {ratio }}=\frac{P_{d y n}}{P_{\text {sta }}}
$$

where $P_{d y n}$ is the power captured from the dynamic simulation and $P_{s t a}$ is the power captured from the static simulation. There are five major variables that significantly impact the response variable, $P_{\text {ratio }}$. These variables are the airfoil shape, the waveform of oscillation, the average angle of attack, the amplitude, and the frequency. An extensive exploration of the airfoil shape and waveform oscillation was performed before running the optimization for the other three variables.

The airfoil selected for the study is the DU 96-W-180 developed at the Delft University of Technology in the Netherlands. An image of its shape is shown in fig. 5. A low camber airfoil was selected because it has a high liftto-drag ratio [24] and its profile data is available online [25]. We also explored high camber airfoils like the S8XX series from the National Renewable Energy Lab (NREL) and the results showed much lower performances.

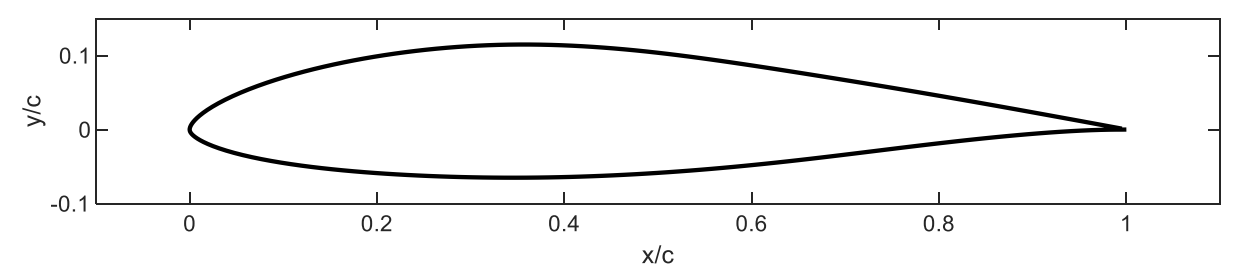

Figure 5: DU 96-W-180 airfoil outline

The waveform selected for the study is a "tilted sinusoid", with an equation of the general form,

$$
\alpha=\sum_{k=1}^{n} \frac{\left(\begin{array}{c}
2 n \\
n-k
\end{array}\right)}{k 2^{2 n-1}} \sin (k 2 \pi f t)
$$

where $f$ is the frequency, $t$ is time, and in general $\left(\begin{array}{c}2 n \\ n-k\end{array}\right)$ is a combination which is defined as, 


$$
\left(\begin{array}{c}
2 n \\
n-k
\end{array}\right)=\frac{(2 n) !}{(n-k) !(n+k) !}
$$

We chose a value of $n=4$ to obtain the desired tilting as shown in fig. 6 . As $n \rightarrow \infty$ the waveform becomes a sawtooth wave.

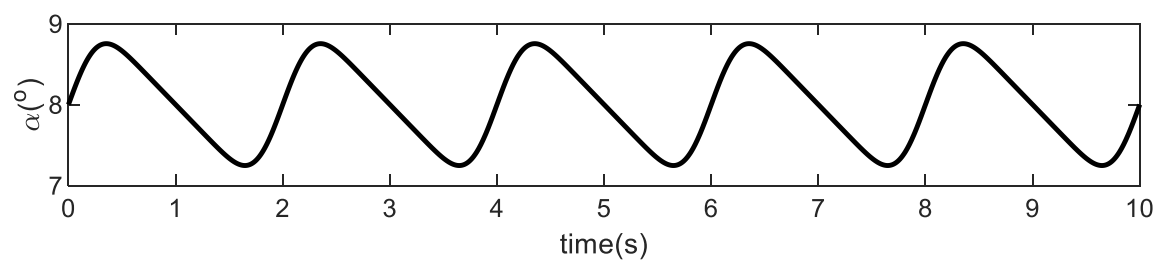

Figure 6: "Tilted sinusoid"

The benefits of a tilted sinusoid oscillation as compared to a regular sinusoid are further described in section 3.1.2 of this paper.

The three independent variables that are changed to form the solution space are the average angle of attack, amplitude, and frequency. The average angle of attack is the center angle that the airfoil oscillates around. The second free variable is the amplitude of the dynamic pitching. The only constraint on this variable is that the amplitude cannot be larger than the average angle of attack to avoid negative angles of attack during the blade's cycle. Negative angles of attack produce very poor or even negative lifts so they will be avoided. The final free variable is the frequency of the oscillation. The major constraint on this variable is that the maximum rotational speed possible with common wind turbine actuators is roughly $10 \mathrm{deg} / \mathrm{s} \mathrm{[26].} \mathrm{One} \mathrm{rotational} \mathrm{cycle} \mathrm{for} \mathrm{the} \mathrm{airfoil}$ is defined as tilting up to the highest angle, then down to the lowest angle, and then back to its starting angle; therefore, the total angular difference the airfoil travels in one cycle is four times the amplitude. Consequently, the equation for maximum possible frequency, $f_{\max }$, is,

$$
f_{\text {max }}=\frac{\omega_{\max }}{4 A}
$$

where $\omega_{\max }$ is the maximum rotational speed of $10 \mathrm{deg} / \mathrm{s}$ and $A$ is the amplitude in degrees. Therefore, the maximum frequency for each simulation case is related to the amplitude of that simulation.

The power ratio values obtained from these simulations are compared to each other for finding the pitching settings that produce the highest efficiency. The static blade simulations are performed in QBlade, the dynamic pitching CFD simulations are performed in Ansys Fluent ${ }^{\circledR}$ and the BEMT calculations are performed in MATLAB ${ }^{\circledR}$.

\subsection{CFD Simulations}

All the CFD Simulations are run with 2D airfoil cross-sections using the SST k- $\omega$ turbulence model as suggested by Menter [27]. The mesh size is initially $0.002 \mathrm{~m}$ for the factorial experiment with refinement around the edge of the airfoil. The mesh size is later enlarged to $0.01 \mathrm{~m}$ for the hill climbing optimization to improve the speed of the simulations after validating that the new mesh size produces accurate results. The larger mesh is shown in fig. 7 . The mesh is coarse far away from the airfoil cross-section but refined in the circular region around the airfoil and even further refined at the leading edge. The average angle of attack is then set by rotating the profile in the geometry setup. Then, a user defined function (UDF) is programmed in $\mathrm{C}$ language to simulate the desired oscillatory movements. After loading the UDF into Ansys Fluent ${ }^{\circledR}$, a $10 \mathrm{~s}$ simulation is run. For the factorial experiment, the time step is $0.002 \mathrm{~s}$ with 5000 time steps but this is increased to a time step of $0.01 \mathrm{~s}$ with 1000 time steps for the hill climbing optimization for the same reason of reducing simulation time. Ansys Fluent ${ }^{\circledR}$ reports the lift, drag, and pitching moment coefficients in the $2 \mathrm{D}$ simulation which can then be plugged into a series of calculations including equations (1) and (2) to calculate the overall power generated by the turbine based on BEMT.

Based on the increase of the mesh size and the time step for the hill climbing optimization to save time, a discrepancy of power ratio of around $2 \%$ is found with respect to the initial simulations. Despite the slight discrepancy between the two meshes and time steps, this is still a valid method for the purpose of finding the optimal point. 


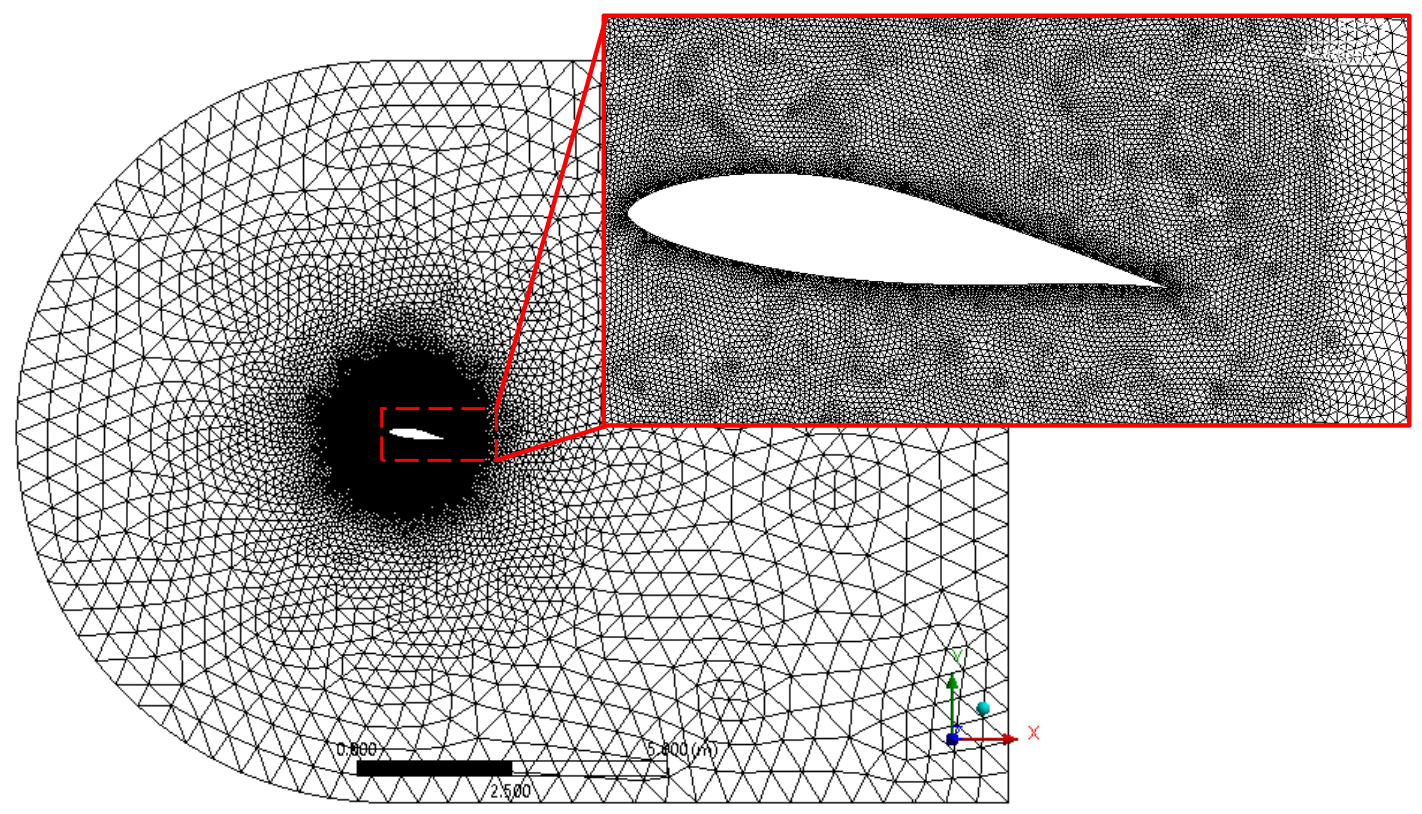

Figure 7: 2D mesh in Ansys Fluent ${ }^{\circledR}$ with detailed view of the mesh around the airfoil

\subsection{Factorial Experiment}

The first portion of the optimization study is based on a factorial experiment. Factorial design is an efficient method for determining the effect of multiple independent variables on a response variable. It involves selecting high and low values for each independent variable to search the solution space and determine the relative effect of each independent variable, or combinations of variables, on the output variable [28]. The only three independent variables that are adjusted are the average angle of attack, the amplitude, and the frequency. A high, low, and medium value are selected for each independent variable. This creates twenty-seven cases that are run in Ansys Fluent®.

\subsection{Hill Climbing Optimization}

The second portion of the optimization study is based on a hill climbing optimization. Hill climbing optimization is an iterative local search technique. For this final solution space search, a greedy approach is used. This means that the algorithm always moves in the direction of higher power ratio with the goal of finding the best solution [29]. Since it is a three-variable optimization, four initial points are required. To find the next prospect point, the centroid of a trapezoid is found, and a new point is projected from the worst point. This new point replaces the worst point from the previous iteration, then a new centroid is found, and a new point projected. This process is iterated until the optimal solution is found. Figure 8 (a), (b) and (c) illustrate the idea for the first three iterations of the optimization. Figure 12 shows the complete optimization path found for this study.

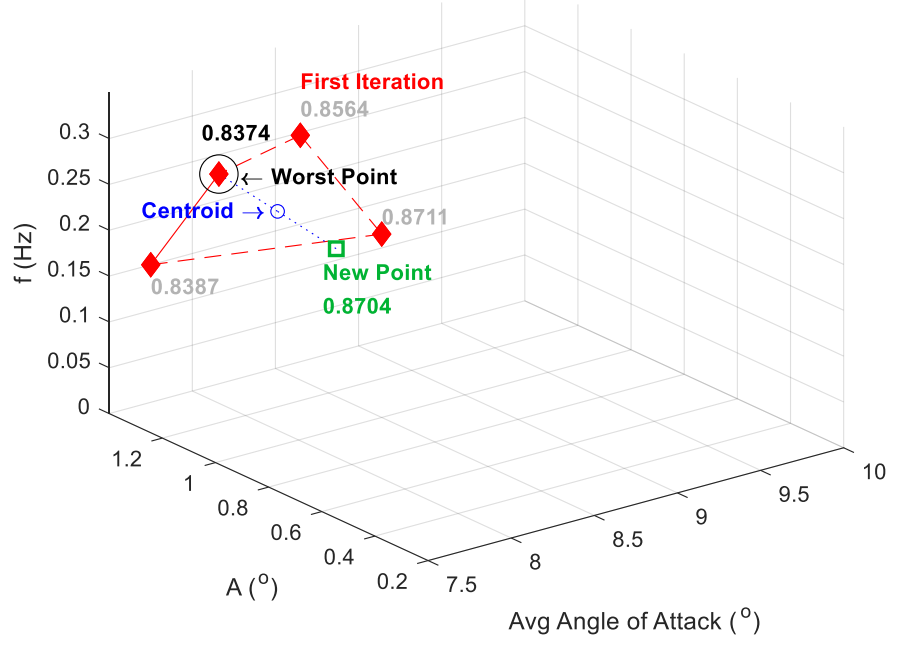

(a)

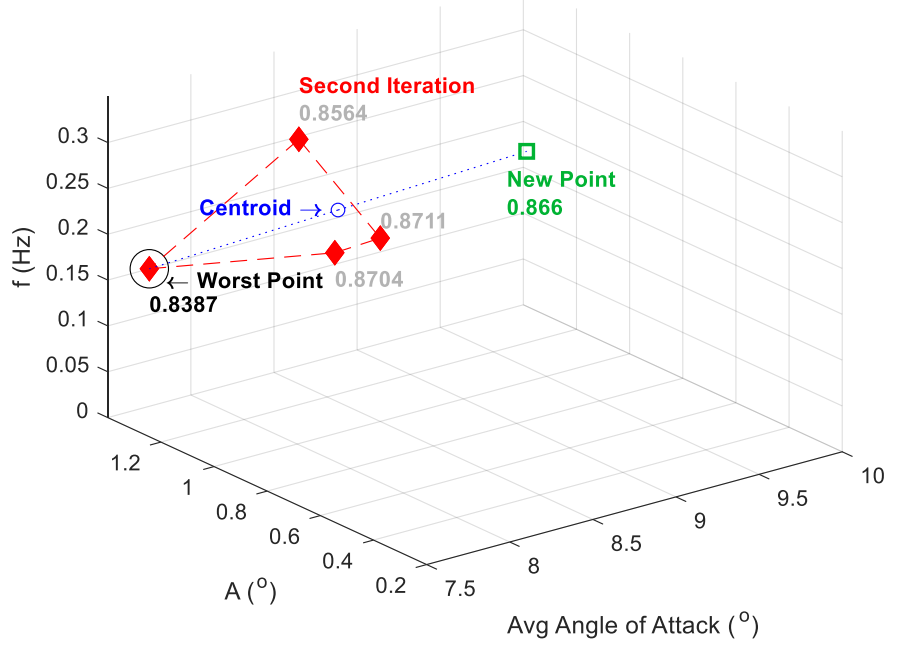

(b) 


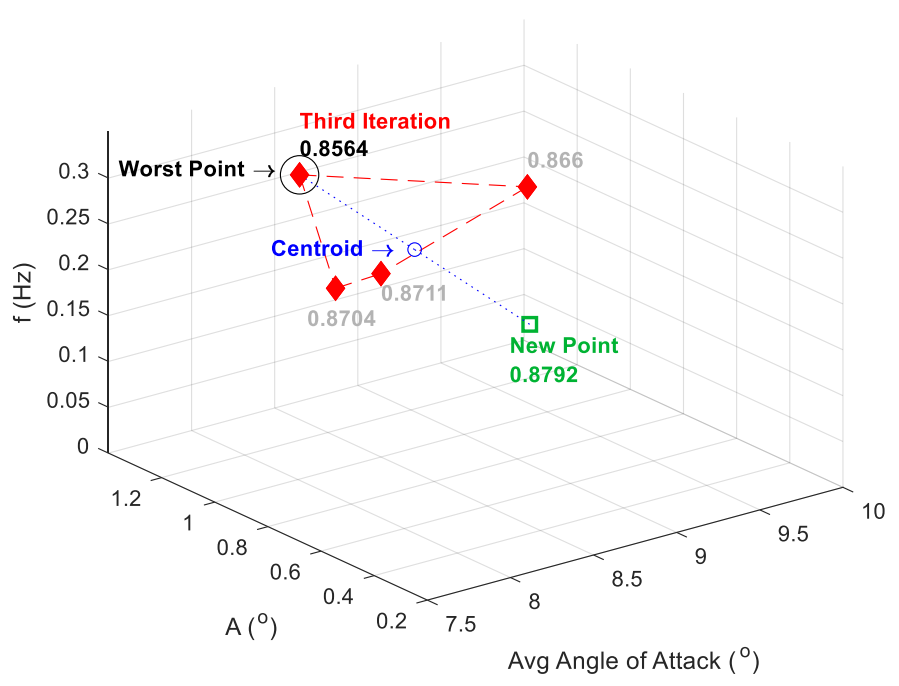

(c)

Figure 8. Step-by-step hill climbing optimization example. The numbers represent the power ratio, $P_{\text {ratio }}$, for the specific average angle of attack, amplitude, and frequency being evaluated.

\section{Results and Discussion}

The results obtained in this work are divided into two. The first set of results are related to the initial CFD simulations used to evaluate the aerodynamic forces acting on the wind turbine blade. The second set of results are related to the optimization results obtained through the factorial experiment and the hill climbing optimization to find the best conditions of oscillation.

\subsection{CFD Simulations}

We explored the effect of the angle of attack on both the lift and drag of airfoils. This information allowed for an educated choice of the solution space to search in the optimization process. Experimental [17] and CFD simulation data of the lift and drag coefficients are shown in fig. 9. Figure 9 (a) shows that the static CFD case, the purple line, exhibits a maximum lift coefficient around an angle of attack of $15^{\circ}$ but in fig. 9 (b) it is clear that the drag can be orders of magnitude larger at this point than it is at low angles of attack. Both the experimental and simulation data on these plots suggest that the optimal combination of lift and drag will probably be found roughly between $5^{\circ}$ and $11^{\circ}$. In that range, the lift coefficient is still high but more importantly, the drag coefficient is extremely low. The data in fig. 9 is from an NREL S809 airfoil but this is still a useful starting range for the airfoil used in the optimization.

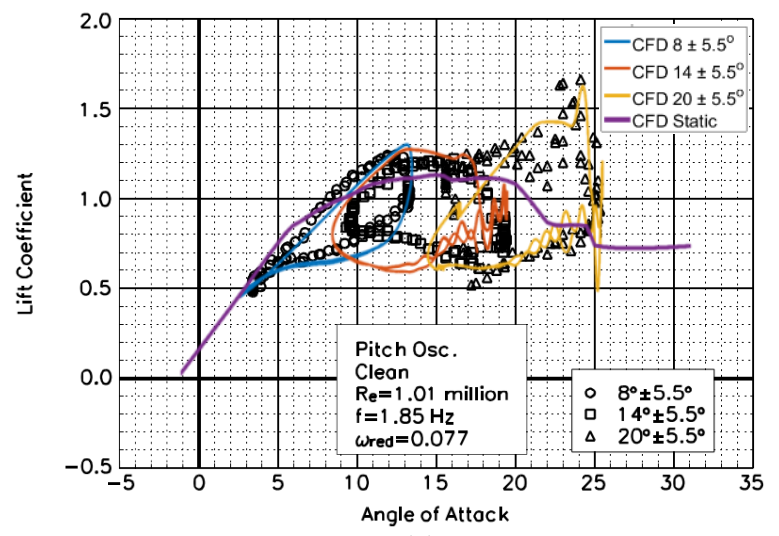

(a)

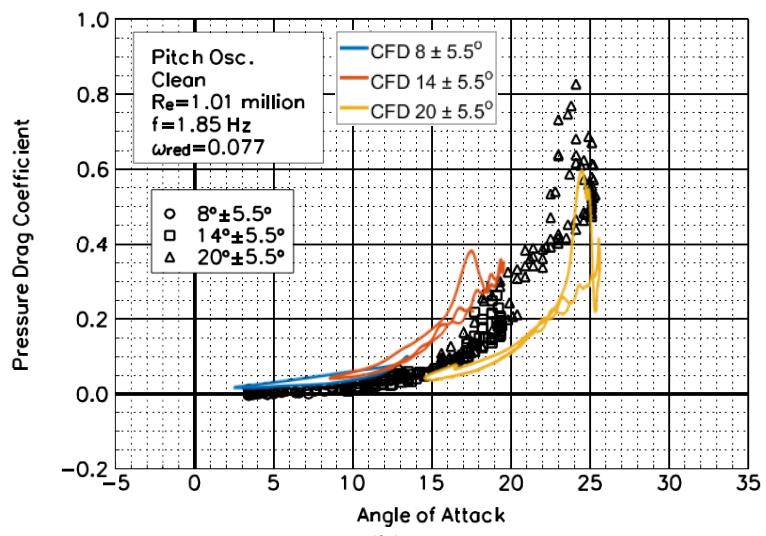

(b)

Figure 9: (a) Dynamic lift coefficients vs. angle of attack curves for S809 airfoil, experimental (black markers) vs CFD data (color lines). (b) Dynamic drag coefficients vs. angle of attack curves for 5809 airfoil, experimental (black markers) vs. CFD data (color lines) 


\subsection{Waveform Evaluation}

Simulation data shows that a tilted sinusoid oscillation waveform performs better than a regular sinusoid. Specifically, a waveform that goes up quickly and down slowly captures more power. A plot of this type of tilted sinusoid is shown in the bottom plot of fig. 10. The data in fig. 10 is from two simulations in Ansys Fluent ${ }^{\circledR}$ with the same conditions but different waveforms. It can be seen in the third plot of fig. 10 that the tilted sinusoid produces higher lift coefficient to drag coefficient ratios than a regular sinusoid. The primary reason for this is because when the airfoil moves down slowly, the lift coefficient stays higher than it does with the regular sinusoid, top plot of fig. 10. The drag coefficient is shown in the second plot of fig. 10; the peak locations differ for the sinusoids, but the average drag coefficient is about the same for both.
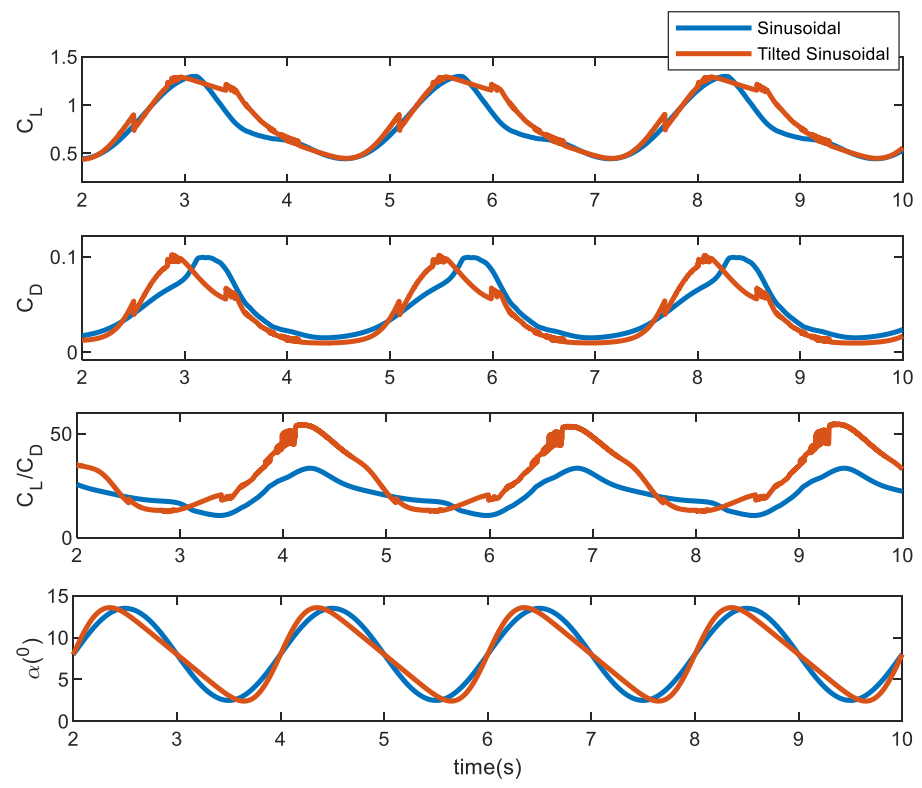

Figure 10: Comparison of $C_{L}$ and $C_{D}$ for regular sinusoid and tilted sinusoid waveforms. Data is from CFD simulations in Ansys Fluent ${ }^{\circledR}$

\subsection{Factorial Experiment}

The only three independent variables that were adjusted were the average angle of attack, the amplitude, and the frequency. A high, low, and medium value were selected for each independent variable. This created twenty-seven cases that were run in Ansys Fluent ${ }^{\circledR}$ and the results are shown in the box diagram in fig. 11. The low, medium, and high values for each independent variable can be seen along the bottom and right edges of the solution space box in the figure and the number at each point is the power ratio value reported from applying BEMT calculations to the data.

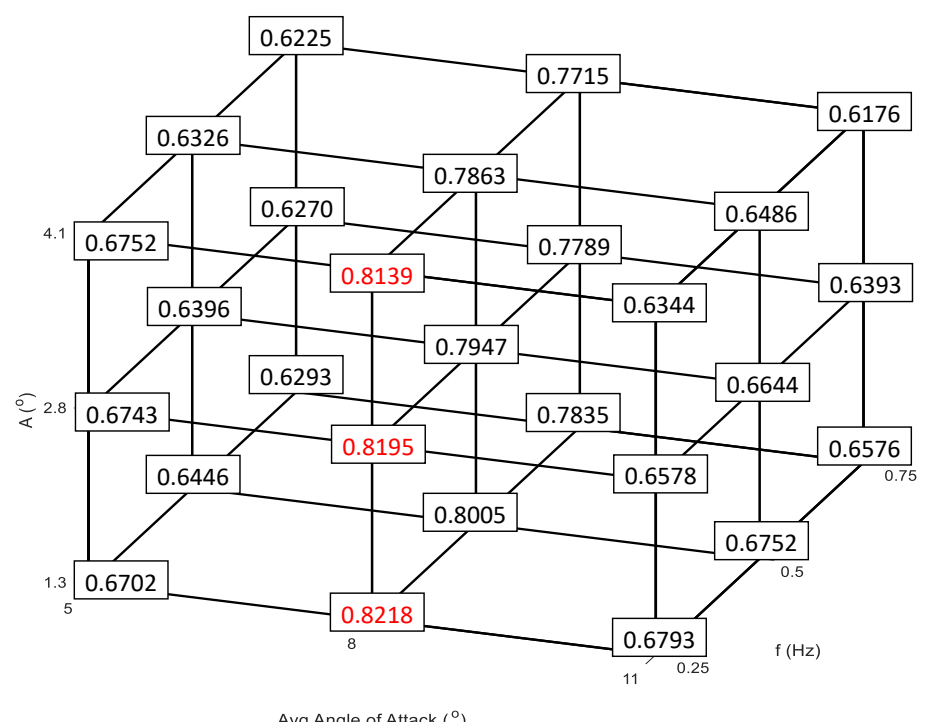

Figure 11: CFD power ratio results from the factorial experiment. Most efficient points highlighted in red 
Figure 11 provides insight into how each variable affects the final power captured from the turbine. The highest power ratio values are highlighted in red. An average angle of attack of 8 degrees captures more power than higher or lower average angles of attack. This is because with an average angle of attack of 8 degrees the oscillation stays in the range of low drag but still has relatively high lift. Also, it appears that lower frequencies produce better power capture; the highest values are found at a frequency of $0.25 \mathrm{~Hz}$. The reason for this mainly being that it requires less input power to pitch the blade at a lower frequency. Finally, amplitude is the variable that has the smallest effect on the power captured by the blade. For the most part, smaller amplitudes result in slightly higher power capture, but the effect is minimal compared to the effect of average angle of attack and frequency. The best result from the initial 27 simulations was used as a starting point for the hill climbing optimization.

\subsection{Hill Climbing Optimization}

The path of the hill climbing optimization is shown in fig. 12. The four starting points are shown as red diamonds, the optimization path is shown as a black solid line and the maximum power ratio is shown as a green star. One of the initial points was the point of highest power ratio from the factorial experiment and the other three were chosen around that with the idea to provide depth in every variable so that the algorithm could search the solution space effectively. A total of twenty-four simulations were run in this portion of the optimization. The search algorithm was reinitialized twice due to stalling and each time it was restarted the size of the search was refined around the highest value from the previous run. The first time the search stalled because the new point had a negative frequency, and the second stall was because the new suggested point had already been simulated. These are both very common problems and solutions for this type of optimization. Overall, a $9 \%$ increase in power capture was discovered with the hill climbing algorithm over the highest power ratio found from the factorial experiment with a final power ratio of 0.8977 . The frequency, average angle of attack and amplitude for the highest power ratio were $0.0065 \mathrm{~Hz}, 8.6022^{\circ}$ and $0.6486^{\circ}$. It is expected to see a power ratio of 1 if the algorithm reaches a frequency of $0 \mathrm{~Hz}$ and an average angle of $8^{\circ}$. However, there is a simulation mismatch that comes from calculating optimal aerodynamic coefficients with QBlade for the static case and with Ansys Fluent ${ }^{\circ}$ for the dynamic case.

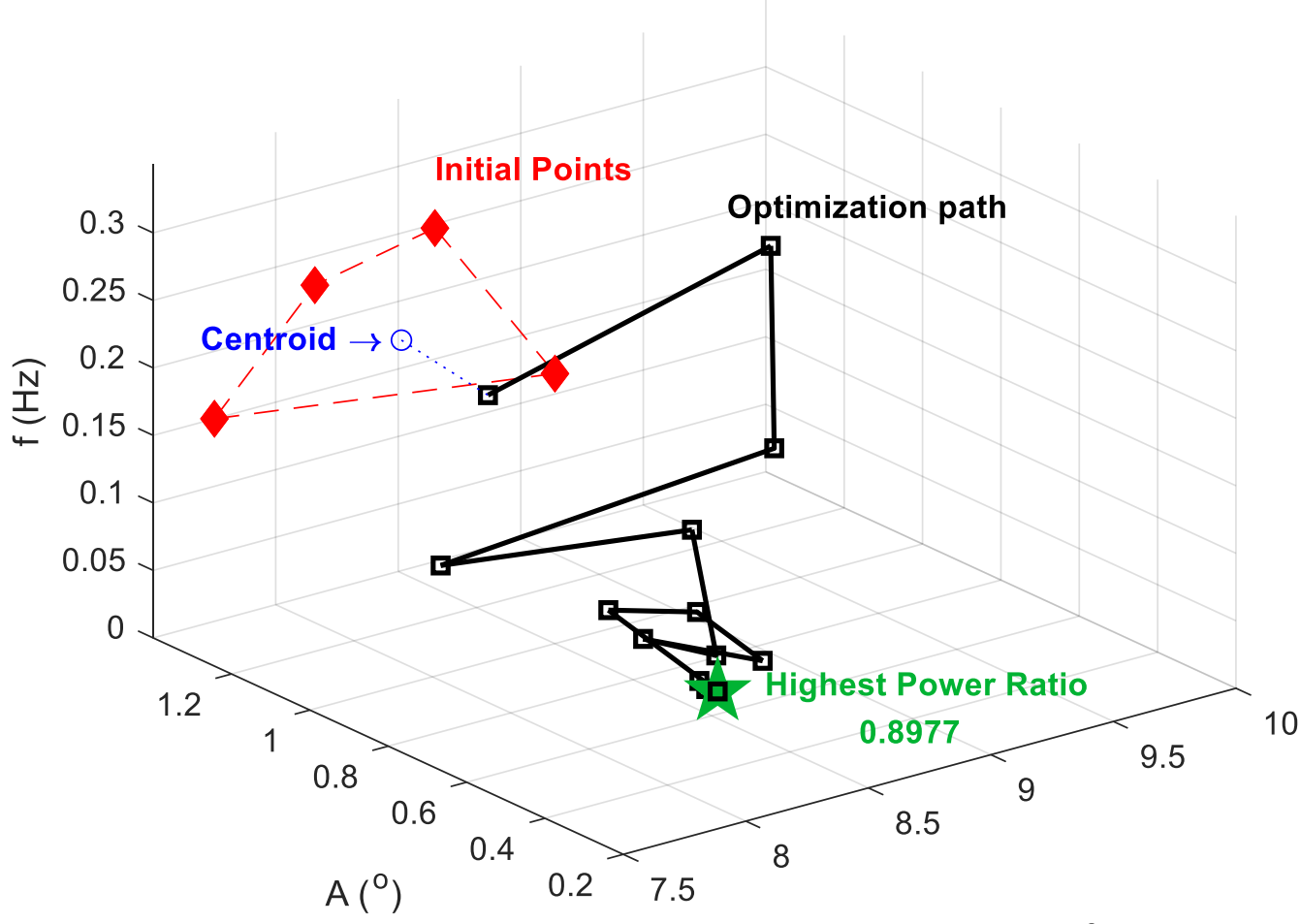

Avg Angle of Attack $\left({ }^{\circ}\right)$

Figure 12: Path of hill climbing optimization shown in black. Initial points shown as red diamonds and point of highest power ratio shown as a green star (The optimization path is only showing the new point for each iteration)

Hill climbing does not guarantee finding the global maximum and it can end up reporting a local maximum as the final result because the greedy approach used cannot climb down hills. This is a general problem of any convex optimization technique [30]. It could get stuck on a local maximum without knowing that there is a higher power ratio value somewhere else in the solution space. A common method for overcoming this is to restart the search numerous times in random locations but there was not enough time to do this because each simulation required 
intensive manual input consisting of determining the new waveform equation, rewriting the waveform code, reinitiating the CFD simulation, and after the simulation, inputting the data into the MATLAB® code to determine the next search point. However, performing the factorial experiment first allowed for a coarse search of the relevant solution space where the maximum power ratio value is almost certain to be found, so it is reasonable to believe that the hill climbing optimization started close to the global maximum. If this is the case, the final point of maximum power ratio found by this optimization can be considered to be the global maximum. Another optimization technique known as ant colony optimization was considered but not used because it also requires a large number of simulations [31].

\section{Conclusion}

We have explored the opportunity to harvest more power from wind by oscillating the pitch of wind turbine blades. The study compared, through simulations, the power captured by static pitch wind turbine blades to the power captured by oscillating the pitch of wind turbine blades. We used CFD analysis combined with BEMT to calculate the power ratio. For the first stage of the study, it was found that oscillating the blades with a tilted sinusoid improved the overall performance when compared to oscillating the blades with a sinusoid wave. This was evident when comparing the lift to drag ratio between the two waveform inputs. For the second stage of the study, an optimization of the oscillating conditions was proposed. The optimization showed that the overall performance of the oscillating case was never higher than the overall performance of the static case. For the optimal conditions, the oscillating blade could only produce $89.77 \%$ of the power produced by the static blade. This is mainly because of the drag forces present in the system and because you have to input extra power into the system to oscillate the blades. 


\section{References}

[1] R Wiser, E Lantz, T Mai, J Zayas, E DeMeo, E Eugeni, J Lin-Powers, R Tusing. Wind Vision: A New Era for Wind Power in the United States. The Electricity Journal, 28(9):120-132, 2015.

[2] U.S. Department of Energy. Wind Vision Report by United States Department of Energy. 2008. [Online]. Available: https://www.energy.gov/eere/wind/wind-vision.

[3] A Betz. Windenergie und Ihre Ausnutzung durch Windmühlen. Vandenhoeck and Ruprecht, Göttingen, 1926.

[4] J F Manwell, J G McGowen, and A L Rogers. Wind Energy Explained: Theory, Design and Application. Wiley, New York, 2009.

[5] R Dutta, F Wang, B F Bohlmann, and K A Stelson. Analysis of Short-Term Energy Storage for Midsize Hydrostatic Wind Turbine. Journal of Dynamic Systems, Measurment and Control, 136(1), 2013.

[6] K E Johnson, L Y Pao, M J Balas, L J Fingersh. Control of Variable-speed Wind Turbines: Standard and Adaptive Techniques for Maximizing Energy Capture. IEEE Control Systems Magazine. 26(3):70-81, 2006.

[7] M A Abdullah, A H Yatim, C W Tan, R Saidur. A review of maximum power point tracking algorithms for wind energy systems. Renewable and sustainable energy reviews. 16(5):3220-7, 2012.

[8] L Y Pao, K E Johnson. A tutorial on the dynamics and control of wind turbines and wind farms. Proc. of the IEEE American Control Conference. 2076-2089, 2009.

[9] F Wang, K A Stelson. Model predictive control for power optimization in a hydrostatic wind turbine. Proc. of the 13th Scandinavian International Conference on Fluid Power. 92:155-160), 2013.

[10] Y Xiao, Y Li, M A Rotea. CART3 field tests for wind turbine region-2 operation with extremum seeking controllers. IEEE Transactions on Control Systems Technology. 27(4):1744-52, 2018.

[11] J Schmitz, N F Diepeveen, N Vatheuer, and H Murrenhoff. Dynamic Transmission Response of a Hydrostatic Transmission Measured on a Test Bench. Proc. of the European Wind Energy Conference and Exhibition (EWEA), 2012.

[12] C Ai, W Gao, Q Hu ,Y Zhang, L Chen, J Guo, Z Han. Application of the Feedback Linearization in Maximum Power Point Tracking Control for Hydraulic Wind Turbine. Energies, 13(6), 2020.

[13] S Sheng. Report on Wind Turbine Subsystem Reliability - A Survey of Various Databases (Presentation), NREL (National Renewable Energy Laboratory). 2013.

[14] B Mohanty, F Wang, and K A Stelson. Design of a Power Regenerative Hydrostatic Wind Turbine Test Platform. JFPS International Journal of Fluid Power System, 11(3):130-135, 2019.

[15] E Mohr, B Mohanty, and K A Stelson. Short-Term Energy Storage System for Hydraulic Hybrid Wind Turbine Transmission. Proc. 2020 Bath/ASME Symposium, Fluid Power Systems Technology, 2020.

[16] A Choudhry, M Arjomandi, and R Kelso. Methods to Control Dynamic Stall for Wind Turbine Applications. Renewable Energy, 86:26-37, 2016. 
[17] M J Hoffmann, R Reuss Ramsay, and G M Gregorek. Effects of Grit Roughness and Pitch Oscillations on the S809 Airfoil. NREL/TP-442-7817, National Renewable Energy Lab, Golden, CO (United States), 1995.

[18] J G Leishman. Challenges in Modeling the Unsteady Aerodynamics of Wind Turbines. ASME 2002 Wind Energy Symposium, 132:141-167, 2002.

[19] J G Holierhoek, J B de Vaal, A H van Zuijlen, and H Bijl. Comparing Different Dynamic Stall Models. Wind Energy, 16:139-158, 2013.

[20] P J Schubel and R J Crossley. Wind Turbine Blade Design. Energies, 5(9):3425-3449, 2012.

[21] E Kulunk. Aerodynamics of Wind Turbines, Fundamental and Advanced Topics in Wind Power. R. Carriveau, 2011.

[22] F Mahmuddin. Rotor Blade Performance Analysis with Blade Element Momentum Theory. Energy Procedia, 105:1123-1129, 2017.

[23] V Dehouck, M Lateb, J Sacheau, and H Fellouah. Application of the Blade Element Momentum Theory to Design Horizontal Axis Wind Turbine Blades. Journal of Solar Energy Engineering, 140:1-9, 2019.

[24] L Prandtl and O G Tietjens. Applied Hydro and Aeromechanics. Dover, New York, 1957.

[25] A M Halawa, B Elhadidi, and S Yoshida. Aerodynamic Performance Enhancement Using Active Flow Control on DU96-W-180 Wind Turbine Airfoil. Evergreen, 5(1):1624, 2018.

[26] M H Hansen, A Hansen, T J Larsen, S Øye, P Sørensen, and P Fuglsang. Control Design for a Pitch-Regulated, Variable Speed Wind Turbine. R-15OO, Ris $\phi$ National Lab, Roskilde, Denmark, 2005.

[27] F R Menter. Two-Equation Eddy-Viscosity Turbulence Models for Engineering Applications. AIAA, 32(8), 1994.

[28] E P George, J S Hunter, W G Hunter, R Bins, K Kirlin IV, and D Carol. Fractional Factorial Designs. Statistics for Experimenters: Design, Innovation, and Discovery. Wiley, New York, 2005.

[29] I P Gent and T W Alsh. Towards an Understanding of Hill-climbing Procedures for SAT. AAAI, 93:28-33, 1993.

[30] D Yuret and M De La Maza. Dynamic Hill Climbing: Overcoming the limitations of optimization techniques. The Second Turkish Symposium on Artificial Intelligence and Neural Networks, 208-212, 1993.

[31] M Dorigo, M Birattari, and S Thomas. Ant Colony Optimization. IEEE Computational Intelligence Magazine, 1(4):28-39, 2006. 